\title{
Nanosecond electric pulses penetrate the nucleus and enhance speckle formation
}

\author{
Nianyong Chen ${ }^{\mathrm{a}, \mathrm{b}, *}$, Allen L. Garner ${ }^{\mathrm{a}, \mathrm{c}, 1}$, George Chen ${ }^{\mathrm{d}}$, Yu Jing ${ }^{\mathrm{e}}$, Yuping Deng ${ }^{\mathrm{e}}$, \\ R. James Swanson a,f, Juergen F. Kolb ${ }^{\text {a,g }}$, Stephen J. Beebe ${ }^{\text {a }}$, Ravindra P. Joshi ${ }^{\text {a,g }}$, \\ Karl H. Schoenbach ${ }^{\mathrm{a}, \mathrm{g}}$ \\ ${ }^{a}$ Frank Reidy Research Center for Bioelectrics, Old Dominion University, Norfolk, VA 23510, USA \\ ${ }^{\mathrm{b}}$ Department of Medicine, Division of Pulmonary and Critical Care Medicine, University of Virginia, Charlottesville, VA 22908, USA \\ ${ }^{c}$ Bioelectromagnetism Laboratory, Department of Nuclear Engineering and Radiological Sciences, University of Michigan, Ann Arbor, MI 48109, USA \\ ${ }^{\mathrm{d}}$ School of Electronics and Computer Science, University of Southampton, Southampton SO17 1BJ, UK \\ e Glennan Center for Geriatrics and Gerontology, Eastern Virginia Medical School, Norfolk, VA 23507, USA \\ ${ }^{\mathrm{f}}$ Department of Biological Sciences, Old Dominion University, Norfolk, VA 23529, USA \\ ${ }^{g}$ Department of Electrical and Computer Engineering, Old Dominion University, Norfolk, VA 23529, USA
}

Received 23 September 2007

Available online 9 October 2007

\begin{abstract}
Nanosecond electric pulses generate nanopores in the interior membranes of cells and modulate cellular functions. Here, we used confocal microscopy and flow cytometry to observe Smith antigen antibody (Y12) binding to nuclear speckles, known as small nuclear ribonucleoprotein particles (snRNPs) or intrachromatin granule clusters (IGCs), in Jurkat cells following one or five $10 \mathrm{~ns}, 150 \mathrm{kV} / \mathrm{cm}$ pulses. Using confocal microscopy and flow cytometry, we observed changes in nuclear speckle labeling that suggested a disruption of pre-messenger RNA splicing mechanisms. Pulse exposure increased the nuclear speckled substructures by $\sim 2.5$-fold above basal levels while the propidium iodide (PI) uptake in pulsed cells was unchanged. The resulting nuclear speckle changes were also cell cycle dependent. These findings suggest that $10 \mathrm{~ns}$ pulses directly influenced nuclear processes, such as the changes in the nuclear RNA-protein complexes.

Published by Elsevier Inc.
\end{abstract}

Keywords: Nucleus; Nuclear speckles; Small nuclear ribonucleoprotein particles/intrachromatin granule clusters; Intracellular electromanipulation; Nanosecond electric pulses

We have been exploring a new nanosecond (ns) time domain in cell biology. When cells are exposed to intense $(50-300 \mathrm{kV} / \mathrm{cm})$ pulsed electric fields (PEFs) with rise times faster than the time it takes for charges on the plasma membrane to redistribute, the electric field can penetrate

\footnotetext{
${ }^{*}$ Corresponding author. Address: Department of Medicine, Division of Pulmonary and Critical Care Medicine, University of Virginia, Charlottesville, VA 22908, USA. Fax: +1 7573142397.

E-mail addresses: nc8u@virginia.edu (N. Chen), kschoenb@odu.edu (K.H. Schoenbach).

${ }^{1}$ Present address: GE Global Research Center, Niskayuna, NY 12309, USA.
}

into the interior of the cell and organelles. If these fields exceed the breakdown threshold of these membranes, nanopores will form in the organelle membranes $[1,2]$. To better understand the mechanism involved, mathematical models for the cellular electropermeabilization due to nsPEFs have been developed $[3,4]$. The resulting changes in cellular structures and functions differ significantly from traditional electroporation [microseconod to millisecond pulsed electric fields (PEFs)] $[1,5,6]$. Increased permeabilization of the plasma membrane, typically demonstrated by measuring propidium iodide (PI) uptake, due to nsPEFs was delayed compared to traditional electroporation $[5,6]$, indicating that it was an indirect effect. These nsPEFs 
induced cellular calcium release $[7,8]$ and apoptosis in cells $[9,10]$ or tumors $[11,12]$. These effects appeared to be due to the charging of interior organelle membranes and the ensuing creation of nanopores. Furthermore, the extremely high electric fields that these pulses induce in intracellular membranes can cause related effects, such as conformational changes of RNA-protein complexes, e.g. nuclear speckles, and induce DNA structural changes, leading to functional modulation of macromolecular structures. Using acridine orange (AO), we showed that 10 or $60 \mathrm{~ns}$ PEFs affected the DNA in the nuclei of HL-60 cells [5]. In addition to the DNA damage observed in Jurkat cells and HL-60 cells exposed to $60 \mathrm{~ns}, 60 \mathrm{kV} / \mathrm{cm}$ pulses [13], other nuclear functions or organization may be impacted by nsPEFs, possibly affecting the RNA-protein complexes and transcriptional functions. However, methodology of the modulation of nuclear processes by nsPEFs remains unknown and further research may open future applications for this technology.

While previous studies with 10 or $60 \mathrm{~ns}$ pulses have demonstrated effects on the nucleus of leukemia cells [5], we further examine the consequence of applying intense $10 \mathrm{~ns}$ pulses on nuclear speckles in Jurkat cells, referred to as small nuclear ribonucleoprotein particles (snRNPs) in G1-phase cells or interchromatin granule clusters (IGCs) in M-phase cells. This study analyzes nuclear speckle responses to intense, $10 \mathrm{~ns}$ pulses in Jurkat cells, possibly leading to changes in the transcriptional sites and functions. We confirmed the hypothesis that charging the nuclear envelope caused nanopore formation in the nuclear envelope and also changes to nuclear structures or subnuclear organizations, such as disrupting pre-messenger RNA splicing mechanisms, resulting in the accumulation of speckles in the nucleus. Analyzing the response of snRNPs by immuno-labeling will clarify how ultrashort pulses cause DNA damage [13] and affect gene expression [14].

\section{Materials and methods}

Cell culture. Jurkat cells, a human T cell leukemia cell line [American Type Culture Collection (ATCC), Manassas, VA], were grown in RPMI1640 medium (phenol red) containing $2 \mathrm{mM}$ L-glutamine (ATCC, VA) and supplemented with $10 \%$ fetal bovine serum (ATCC, VA), $100 \mathrm{U} / \mathrm{ml}$ penicillin, and $100 \mu \mathrm{g} / \mathrm{ml}$ streptomycin (Sigma, St. Louis, MO), and were maintained at $37{ }^{\circ} \mathrm{C}$ with $5 \% \mathrm{CO}_{2}$. The cells used in our experiments ranged between 30 and 50 passages. We verified that cell viability exceeded $90 \%$ daily and prior to all experiments by using trypan blue exclusion (TBE) $(0.4 \%)$ (Sigma).

Cell synchronization and cell cycle analysis. Jurkat cells were plated at a density of $2.0 \times 10^{6}$ cells $/ 10 \mathrm{ml}$ fresh RPMI-1640 medium, as described above, in a $100-\mathrm{mm}$ tissue culture plate (Corning Inc.) and incubated overnight at $37{ }^{\circ} \mathrm{C}$ with $5 \% \mathrm{CO}_{2}$. Plates were incubated for an additional $24 \mathrm{~h}$ in the presence of $40 \mathrm{ng} / \mathrm{ml}$ nocodazole (Sigma) or $500 \mu \mathrm{M}$ mimosine (Sigma). Thereafter, the synchronized cells were washed once and fixed before pulse application and at designated time points post-pulse in icecold $100 \%$ ethanol for $30 \mathrm{~min}$. Fixed cells were underplated with $500 \mu \mathrm{l}$ of ice-cold fetal bovine serum and spun at $1000 \mathrm{rpm}$ for $5 \mathrm{~min}$. Cells were then washed in $1 \mathrm{ml}$ PBS and spun at $1000 \mathrm{rpm}$ for $5 \mathrm{~min}$. RNase A $(125 \mu \mathrm{l})$ (Quiagen) was added to the cell pellets and incubated at $37^{\circ} \mathrm{C}$ for $15 \mathrm{~min}$. We added $125 \mu \mathrm{l}$ of propidium iodide (PI) $(62.5 \mu \mathrm{g})$ and incubated the cells at room temperature for 30 min prior to flow cytometry analysis. Cell samples were examined by flow cytometry (FACS Calibur) using CellQuest Software with data analysis conducted using ModfitLT software.

Administration of the intense nsPEFs. Synchronized Jurkat cells were examined before pulse application and at designated time points postpulse by flow cytometry. We next loaded $450 \mu$ of Jurkat cell suspension $\left(1 \times 10^{6}\right.$ cells $)$ containing the synchronizing drug into gene pulser ${ }^{\circledR}$ cuvettes (Bio-Rad) with an electrode distance of $2 \mathrm{~mm}$. The synchronized cell suspension was exposed to one or five $10 \mathrm{~ns}, 150 \mathrm{kV} / \mathrm{cm}$ pulses with riseand fall-times of $1-2 \mathrm{~ns}$. The pulses were generated by a Blumlein pulse generator with an impedance of $10 \mathrm{Ohm}$. The Blumlein generator consists of two transmission lines with equal length and impedance in series with the load impedance matching the overall impedance of the two transmission lines, $2 Z_{0}$. More details can be found elsewhere [15].

The pulsed Jurkat cell suspensions containing the synchronizing drug were continually incubated at $37{ }^{\circ} \mathrm{C}$ in $5 \% \mathrm{CO}_{2}$. Cell viability was measured by TBE before pulsing and $10 \mathrm{~min}, 1 \mathrm{~h}$, and $3 \mathrm{~h}$ after the pulse(s). Plasma membrane permeability was examined by measuring PI uptake post-pulse [5].

Nuclear speckles labeling by immunocytochemistry. Synchronized Jurkat cells at designated times post-pulse were washed once in PBS before fixation. Cells were then fixed with $4 \%$ formaldehyde in cytoskeletal buffer [CSK, $10 \mathrm{mM}$ Pipes, pH 6.8, $300 \mathrm{mM}$ sucrose, $100 \mathrm{mM} \mathrm{NaCl}, 3 \mathrm{mM}$ $\mathrm{MgCl}_{2}$, and $1 \mathrm{mM}$ EGTA, (Sigma)] for $30 \mathrm{~min}$. To improve antibody penetration, the pulsed Jurkat cells were permeabilized by using $0.5 \%$ Triton X-100 in CSK for $10 \mathrm{~min}$. Nonspecific binding sites were blocked with TBS-I buffer $[10 \mathrm{mM}$ Tris, $\mathrm{pH} 7.7,150 \mathrm{mM} \mathrm{NaCl}, 3 \mathrm{mM} \mathrm{KCl}$, $1.5 \mathrm{mM} \mathrm{MgCl} 2,0.05 \% \mathrm{vol} / \mathrm{vol}$ Tween $20,0.1 \% \mathrm{BSA}, 0.2 \% \mathrm{wt} / \mathrm{vol}$ glycine (Sigma)] for $1 \mathrm{~h}$. Y12 antibody (Abcam, Inc., MA) was diluted in TBS-I to a 1:50 ratio and added to the cells, which were then incubated at $4{ }^{\circ} \mathrm{C}$ for $1 \mathrm{~h}$. To remove unbound antibodies, cells were rinsed three times in PBS with $0.05 \%$ Tween 20 . The cells were then incubated with the second antibody, 1:50 fluorescein (FITC) conjugated goat anti-mouse IgG (Jackson ImmunoResearch Laboratories, Inc., PA), at $4{ }^{\circ} \mathrm{C}$ for $1 \mathrm{~h}$. After washing once, cell samples were passed through flow cytometry, and coverslips were also mounted on slides for imaging by confocal microscopy.

Determination of nuclear speckles by flow cytometry. As described above, the synchronized cells in M or G1 phase $\left(1.5 \times 10^{4}\right.$ cells) labeled with Y12 antibody specific to nuclear speckles were acquired by using FACS Calibur (Becton-Dickinson) flow cytometry. Data were analyzed by CellQuest software for examining speckled cells.

Detection of nuclear speckles by confocal microscopy. Jurkat cells synchronized to the M or G1 phase were exposed to $10 \mathrm{~ns}, 150 \mathrm{kV} / \mathrm{cm}$ pulses and labeled with Y12 antibody specific to nuclear speckles at different time points after exposure. Fifty thousand cells in $100 \mu \mathrm{lBS}$, labeled with Y12 and FITC, were mounted and dried on slides with a Prolong ${ }^{\circledR}$ Antifade Kit (Molecular Probes) for detecting the nuclear speckles by confocal microscopy. Data were collected using an Olympus Fluoview FW 300 confocal scanning laser fluorescent microscope system (Japan) with high contrast and highly improved resolution in the light axis direction, as previously described [5].

Statistical analysis. We used SPSS Version 11.0 for Windows for statistical analysis and Student's $t$-test to calculate the variance between the speckles in pulsed and unpulsed cells. Differences were considered statistically significant when $P<0.05$. Data are presented as means $\pm \mathrm{SEM}$.

\section{Results}

Using immune labeling, temporally, and spatially resolved images of nuclear speckles generated by $10 \mathrm{~ns}$ pulse-induced stimulation were measured by recording intra-nuclear fluorescence of Jurkat cells caused by the binding of Y12 antibody with FITC to the nuclear speckles. We examined the nuclear speckles before the pulse and at 10 , 
60, and 180 min post-pulse. Nuclear speckles were detected after pulsing by examining the cellular fluorescence corresponding to the predicted molecular weight of Smith antigen (Sm) using flow cytometry and confocal microscopy. To assess cell cycle dependence, we synchronized the Jurkat cells to $\mathrm{M}$ or G1 phase and quantitatively examined the nuclear speckles in each phase by flow cytometry or confocal microscopy, respectively. Applying one or five $10 \mathrm{~ns}, 150 \mathrm{kV} / \mathrm{cm}$ pulse(s) caused no PI uptake in all experiments.

\section{Nanosecond pulses alter the nuclear speckles in mitotic phase cells}

To obtain the mitotic cells for analyzing the nuclear speckles, we used nocodazole to synchronize the Jurkat cells in $\mathrm{M}$ phase before pulse application. Nocodazole blocks cell cycle progression in the G2-M phase by disrupting the mitotic spindle [16]. After $24 \mathrm{~h}$ of culturing with $40 \mathrm{ng} / \mathrm{ml}$ nocodazole, $95 \%$ of Jurkat cells were arrested in $\mathrm{M}$ phase for all pulsed experiments. Applying a $10 \mathrm{ns,}$ $150 \mathrm{kV} / \mathrm{cm}$ pulse to these synchronized Jurkat cells linked the Y12 antibody to Smith antigen in nuclear speckles. The number of speckles decreased immediately following exposure then increased $3 \mathrm{~h}$ later compared to the unpulsed cells (Fig. 1A). This suggests that nsPEFs induce functional and conformational changes in nuclear subunits, such as speckles, probably decreasing the number of transcription factors in Jurkat cells within $1 \mathrm{~h}$. However, exposing cells in the mitotic phase to five consecutive $10 \mathrm{~ns}$ pulses immediately and significantly increased the number of nuclear speckled substructures. Fig. 1B shows the increased FITC-fluorescence intensity in cells with Y12 antibody bound to the speckled protein complexes. Applying five pulses accelerated IGC formation, suggesting a cumulative effect on stimulating nuclear speckled structure reorganization and showing that applying more pulses promotes rapid speckle formation.

Morphological changes in the nuclear speckled substructures exposed to nsPEFs were significant and striking. While applying $10 \mathrm{~ns}, 150 \mathrm{kV} / \mathrm{cm}$ pulses did not alter cell viability by TBE, it caused FITC uptake to increase in the M-phase cells during our measurements $(3 \mathrm{~h}$ ) compared to the unexposed M-phase Jurkat cells. Applying five consecutive $10 \mathrm{~ns}, 150 \mathrm{kV} / \mathrm{cm}$ pulses caused a large fraction of the pulsed cells $(90 \%)$ to bind Y12 antibodies. This also showed that FITC linked to the nuclear speckled structures in cells at $3 \mathrm{~h}$ post-pulse (Fig. 2A). Only $20 \%$ of control cells displayed FITC-fluorescence within $3 \mathrm{~h}$, suggesting that nsPEFs stimulated nuclear speckle reorganization and substructure formation. Using Y12 antibody detection, nuclear speckles appeared as spots in the nucleus of synchronized Jurkat cells in the M-phase of unpulsed cells. Pulse exposure increased the size and number of speckled subunits in the M-phase cells $10 \mathrm{~min}$ and $1 \mathrm{~h}$ after exposure and created diffuse structures with bright fluorescence throughout the nucleoplasm $3 \mathrm{~h}$ post-pulse, as viewed by confocal microscopy (Fig. 2B). This demonstrated that
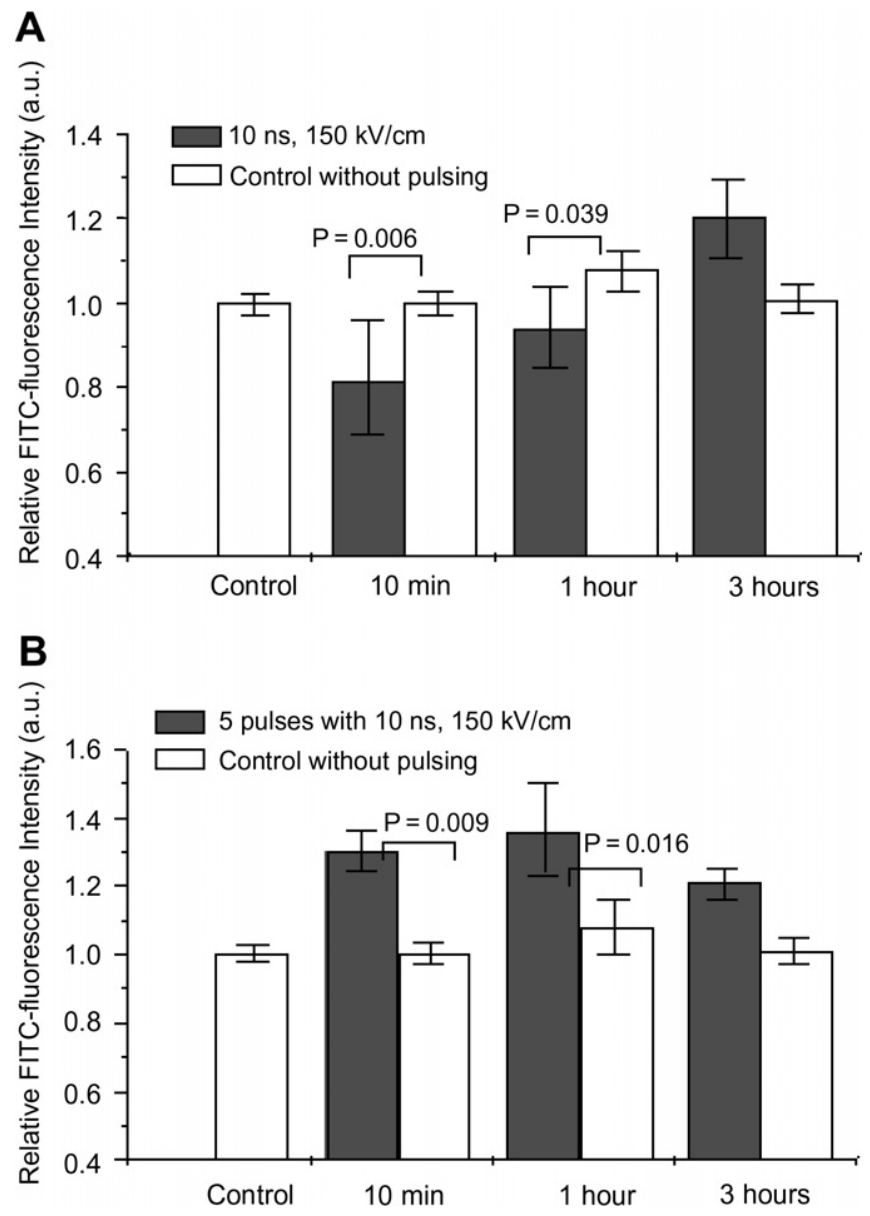

Fig. 1. Flow cytometric quantitative analysis in the neuclear speckle changes in M-phase Jurkat cells at 10, 60, and 180 min after the pulse(s) of $10 \mathrm{~ns}, 150 \mathrm{kV} / \mathrm{cm}$. Nuclear speckles were detected by intracellular immuno-labeling with Y12 antibody, a specific nuclear speckle marker, in Jurkat cells. Ninety-five percent of Jurkat cells were synchronized at M phase by nocodazole treatment for $24 \mathrm{~h}$. (A) A single pulse, (B) five consecutive pulses. $P$ value by Student's test, two-tail of the log converted values. Error bars are calculated using standard deviation.

IGC reorganization and accumulation was stimulated in the mitotic cells, and, in fact, these nuclear RNA-protein complexes showed conformational changes after pulse application with this protocol.

\section{Nanosecond pulses accelerate the aggregation of nuclear speckles in interphase cells}

We applied mimosine to block Jurkat cells at the early synthesis phase because this chemically arrested the cells in G1 phase before DNA replication [17]. Seventy percent of Jurkat cells were arrested at the G1 phase by treating them with $500 \mu \mathrm{M}$ mimosine for $24 \mathrm{~h}$ prior to pulse application. When Jurkat cells were synchronized in the G1 phase with mimosine and exposed to a $10 \mathrm{~ns}, 150 \mathrm{kV} / \mathrm{cm}$ pulse, their nuclear speckled fluorescence differed from that of unexposed cells. Quantitative flow cytometric analysis (Fig. 3A) showed the temporal decrease in cells with high FITC-fluorescence after $10 \mathrm{~min}$. This was followed by an 

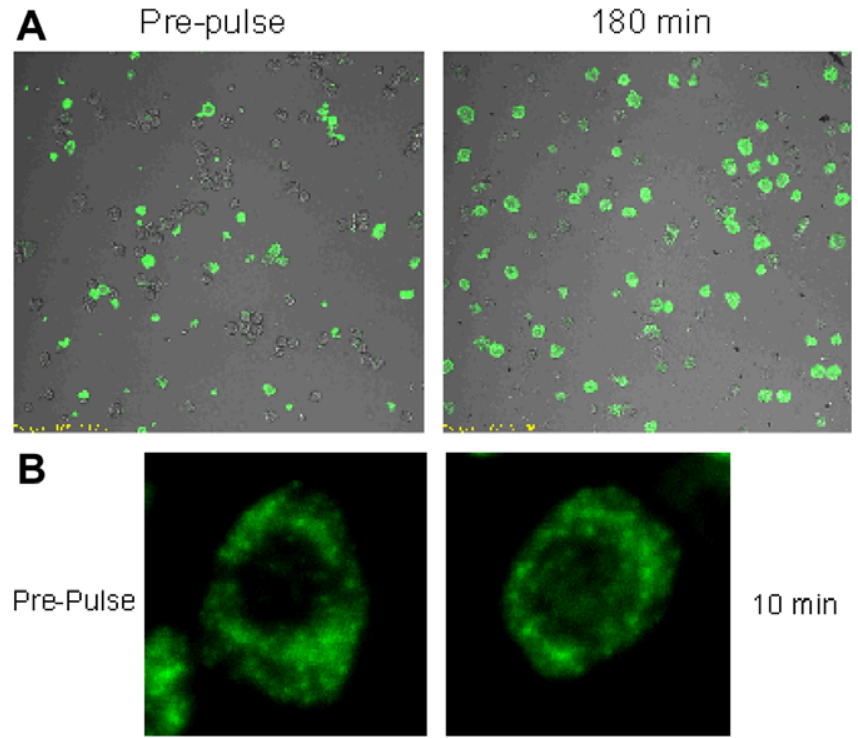

$10 \mathrm{~min}$
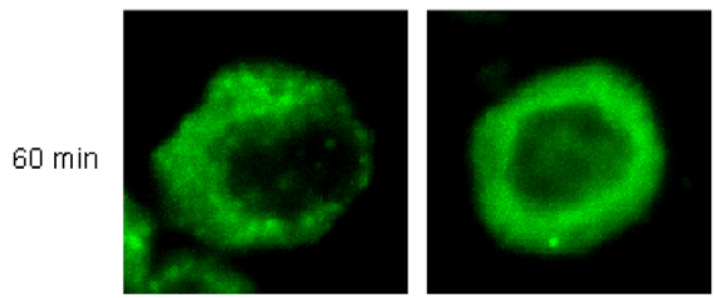

Fig. 2. (A) Representative confocal microscopic images of Y12 antibody binding to speckles in M-phase Jurkat cells unexposed (left) and $3 \mathrm{~h}$ (right) after five consecutive pulses with $10 \mathrm{~ns}$ duration, $150 \mathrm{kV} / \mathrm{cm}$ electric field (400×, merging image with FITC-Y12 uptake and brightfield illumination); (B) Typical confocal microscopic three-dimensional images of Y12 antibody binding to speckles in a Jurkat cell in $\mathrm{M}$ phase before, at $10 \mathrm{~min}$, $1 \mathrm{~h}$ and $3 \mathrm{~h}$ after five consecutive $10 \mathrm{~ns}, 150 \mathrm{kV} / \mathrm{cm}$ pulses.

increase, with a possible delay in speckle function in G1 cells with high fluorescence, $3 \mathrm{~h}$ after the pulse. However, applying five $10 \mathrm{~ns}$ pulses with the same electric field enhanced the speed and quantity of snRNP formation (Fig. 3B). Compared with the control, the relative FITCfluorescence intensity increased 2.5 -fold in the nuclear speckled RNA-protein particles in G1 cells $1 \mathrm{~h}$ after five consecutive $10 \mathrm{~ns}, 150 \mathrm{kV} / \mathrm{cm}$ pulses. This also suggests that nsPEF-induced speckled substructure aggregation is a cumulative effect.

After applying five consecutive $10 \mathrm{~ns}, 150 \mathrm{kV} / \mathrm{cm}$ pulses, the exposed cells with bright FITC-fluorescence demonstrated Y12 antibody binding to the nuclear speckled RNA-protein components in all pulsed cells $3 \mathrm{~h}$ post-pulse. In the unpulsed control, less than $10 \%$ of cells with FITCfluorescence were detected on confocal microscopic images by overlapping the bright field and FITC image (Fig. 4A). Using confocal microscopy, images were acquired in multiple sections. The nuclear speckle structures were observed as clouds and increased in both size and number. They aggregated in the nucleus of Jurkat cells in G1 phase after applying five consecutive $10 \mathrm{~ns}, 150 \mathrm{kV} / \mathrm{cm}$ pulses (Fig. 4B). These findings implied that the RNA-protein complexes in the nucleus accumulated rapidly in interphase
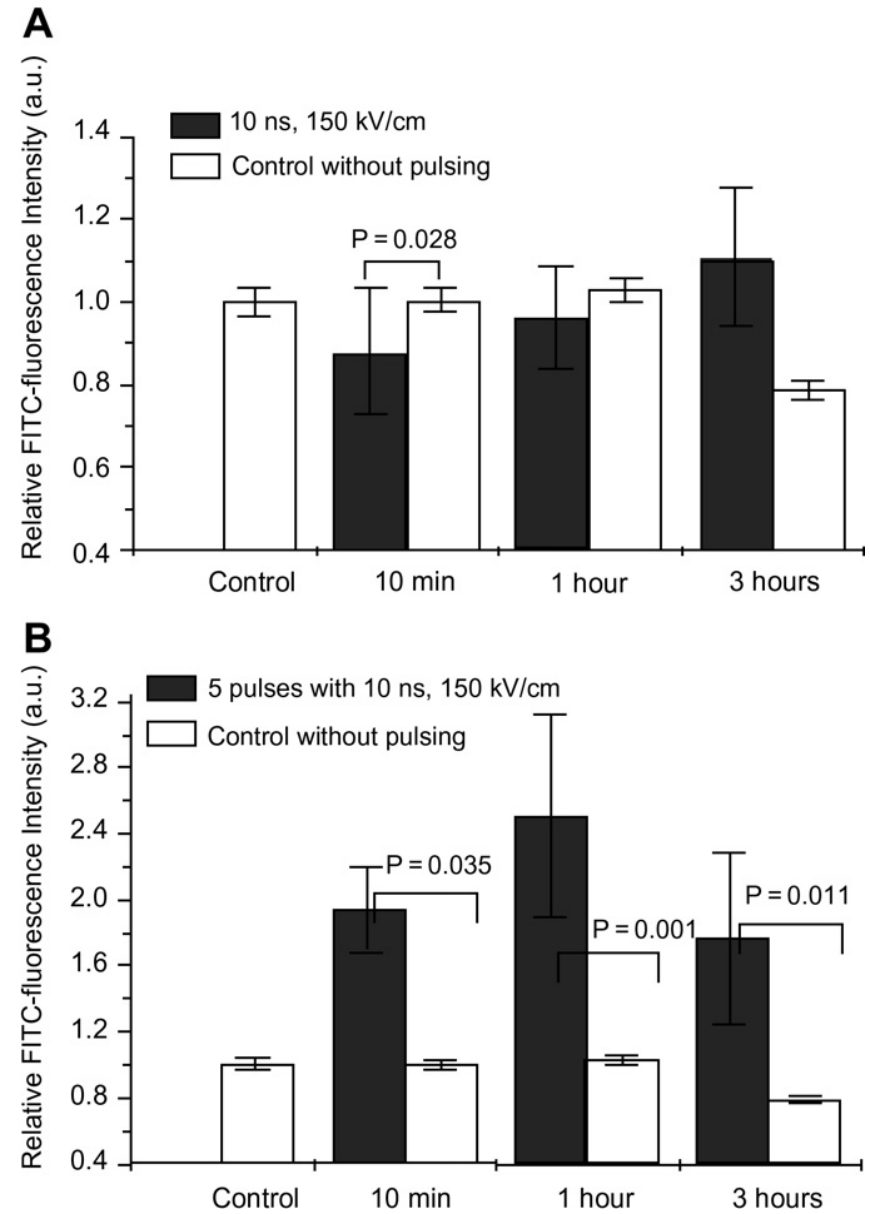

Fig. 3. Nuclear speckle changes in Jurkat cells at G1 phase by flow cytometric quantitative analysis using Y12 antibody by applying $10 \mathrm{~ns}$ pulse(s) with a $150 \mathrm{kV} / \mathrm{cm}$ electric field. Seventy percent Jurkat cells were synchronized by mimosine for $24 \mathrm{~h}$ before pulsing in the pulsed protocol. $P$ values by Student's test, two-tail of the log convert values. (A) A single pulse, (B) five consecutive pulses. Error bars are calculated using standard deviation.

Jurkat cells after exposure and showed the impact of repeated pulses.

\section{Discussion}

Compared to traditional electroporation, nsPEFs preferentially charge the membranes of subcellular organelles, thereby inducing distinct effects on cellular structure and function that are predominantly intracellular in nature $[1,2,5,8]$. The resulting delayed plasma membrane permeabilization was likely secondary, arising due to subcellular effects [2] rather than direct electroporation $[18,19]$. In previous confocal microscopic real time studies, we compared the nuclear and plasma membrane effects of 10 and $60 \mathrm{~ns}$ pulses of approximately the same energy [5]. We interpreted the specific changes of nuclear materials by AO fluorescence as arising due to charging of the nuclear envelope. They could also arise due to the interference of high electric fields generated by nuclear envelope charging with nuclear function, resulting in AO intercalation changes in DNA [5]. 

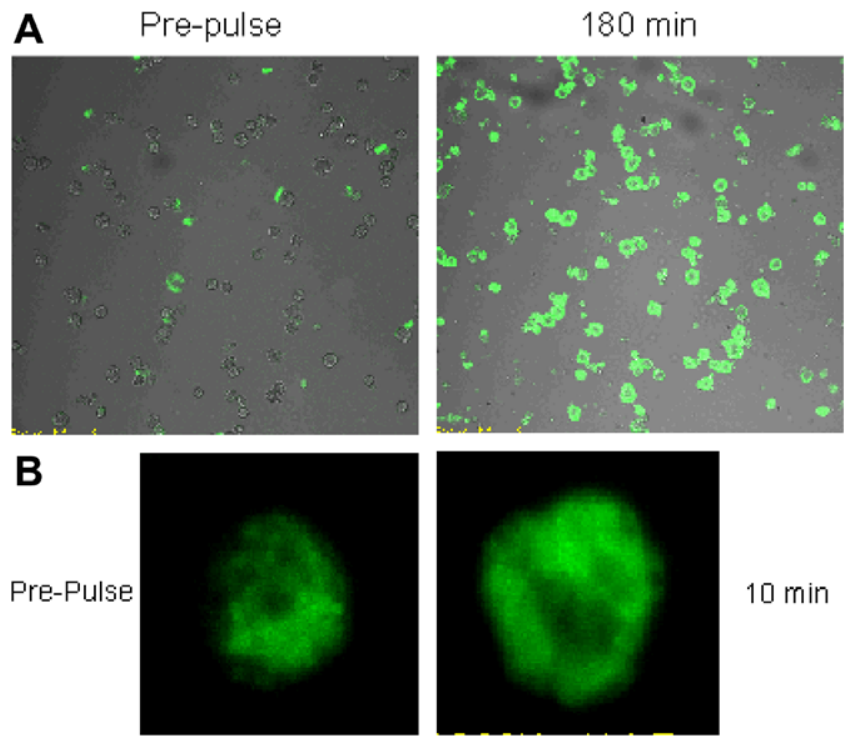

$10 \mathrm{~min}$
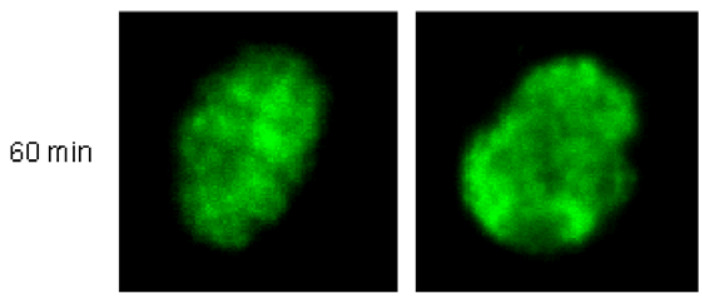

$180 \mathrm{~min}$

Fig. 4. (A) Representative confocal microscopic images of Y12 antibody binding to speckles in G1-phase Jurkat cells unexposed (left) and $180 \mathrm{~min}$ (right) after five $10 \mathrm{~ns}, 150 \mathrm{kV} / \mathrm{cm}$ pulses. (B) Confocal microscopic threedimensional images of the snRNPs in a Jurkat cell in G1 phase before, at 10,60 , and $180 \mathrm{~min}$ after five consecutive $10 \mathrm{~ns}, 150 \mathrm{kV} / \mathrm{cm}$ pulses. Inmunolabeling used Y12 antibody binding to nuclear speckles.

In other studies, nsPEFs induced DNA damage that was most likely a direct effect of the pulses [13] and affected cell survival, clearly indicating that they can affect the nuclear structures $[5,13]$. However, it remains unknown whether nsPEFs might affect other charged nuclear functional substructures. Since DNA is a heavily charged intracellular component whose folded spiral structures are distributed close to the nuclear envelope, we postulated that it may be susceptible to nsPEFs [13].

In this study, we demonstrated that nsPEFs affected nuclear speckles by disrupting or reorganizing the snRNP formation in Jurkat cells. We further observed that this interaction was cell cycle dependent. These changes in nuclear RNA-protein complex components probably reflect a disruption of RNA transcription mechanisms, thus supporting our hypothesis. The results demonstrate that $10 \mathrm{~ns}$ pulses, which are shorter than the charging time of the plasma membrane, altered sub-nuclear structures, perhaps by inhibiting the RNA transcription mechanisms resulting in the accumulation of snRNPs. Furthermore, by using consecutive nsPEFs, it may be possible to modulate nuclear functions for controlling the RNA transcription and gene expression mechanisms in the nucleus. This study shows for the first time that nsPEFs altered nucleoplasm-protein complexes. These observed functional mod- ifications in speckled RNA-protein complexes with Y12 antibodies as a specific speckle marker with FITC-fluorescence, could be explained by the electric field generated in the boundary layer adjacent to the nuclear envelope becoming sufficiently large to cause conformational changes in RNA-protein complexes.

Nuclear speckles were tagged with the Y12 monoclonal antibody $(\mathrm{mAb})$ to Smith antigen $(\mathrm{Sm})$, which recognizes common core proteins of snRNPs involved in RNA processing $[20,21]$. The Smith antigen precipitates the small nuclear RNA, providing direct evidence that the Smith antigen resides on RNA-protein complexes [20]. The nuclear speckles are rich in pre-mRNA splicing factors and are localized in the interchromatin regions in the nucleoplasm. These nuclear subunits are dynamic groups of RNA and proteins, appearing as irregular, punctate structures that vary in size and shape. These speckles functioned as assembly compartments that supplied splicing factors to active transcription sites. Cell studies showed that the splicing factors are recruited from speckles to transcription sites [22]. Conversely, when transcription [23] or pre-mRNA splicing [24] are inhibited, splicing factors accumulate in enlarged, rounded speckles. The distribution of speckled substructures can directly reflect the nuclear functional changes, affecting active transcription sites and gene expression. In this case, nsPEFs caused speckled subunit accumulation, possibly indicating that nsPEFs inhibited the active transcription sites that are related to gene expression with the RNA splicing factors accumulating predominantly in enlarged, aggregated speckled structures. The nsPEFs may also suppress cell growth. Enhanced gene expression with active transcription sites generates abnormal proteins, which can then stimulate cell proliferation. The cells were not destroyed within our protocol's time span but there may be a functional change that is currently being investigated.

\section{Acknowledgments}

This work was supported in part by an Air Force Office of Scientific Research (AFOSR) MURI grant on Subcellular Response to Narrow Band and Wide Band Radio Frequency Radiation, administered by Old Dominion University. One author (A.L.G.) was supported by a National Defense Science and Engineering Graduate Fellowship sponsored by the Department of Defense.

\section{References}

[1] K.H. Schoenbach, S.J. Beebe, E.S. Buescher, Intracellular effect of ultrshort electrical pulses, Bioelectromagnetics 22 (2001) 440-448.

[2] K.H. Schoenbach, R.P. Joshi, J. Kolb, N. Chen, M. Stacey, P. Blackmore, E.S. Buescher, S.J. Beebe, Ultrashort electrical pulses open a new gateway into biological cells, Proc. IEEE 92 (2004) 1122 1137.

[3] R.P. Joshi, Q. Hu, R. Aly, K.H. Schoenbach, Self-consistent simulations of electroporation dynamics in biological cells subjected to ultrashort electrical pulses, Phys. Rev. E 64 (2000) 1901-1913. 
[4] R.P. Joshi, Q. Hu, K.H. Schoenbach, Modeling studies of cell response to high-intensity electric fields-implications of for intracellular manipulation, IEEE Trans. Plasma Sci. 32 (2004) 1477-1686.

[5] N. Chen, K.H. Schoenbach, J.F. Kolb, R.J. Swanson, A.L. Garner, J. Yang, R.P. Joshi, S.J. Beebe, Leukemic cell responses to nanosecond electric pulses, Biochem. Biophys. Res. Commun. 317 (2004) 421-427.

[6] J. Deng, K.H. Schoenbach, E.S. Buescher, P.S. Hair, P.M. Fox, S.J. Beebe, The effects of intense submicrosecond electrical pulses on cells, Biophys. J. 84 (2003) 2709-2714.

[7] P.T. Vernier, Y. Sun, L. Marcu, S. Salemi, C.M. Craft, M.A. Gundersen, Calcium bursts induced by nanosecond electric field, Biochem. Biophys. Res. Commun. 310 (2003) 286-295.

[8] J.A. White, P.F. Blackmore, K.H. Schoenbach, S.J. Beebe, Stimulation of capacitative calcium entry in HL-60 cells by nanosecond pulsed electric fields, J. Biol. Chem. 279 (2004) 22964-22972.

[9] S.J. Beebe, P.M. Fox, L.J. Rec, K. Somers, R.H. Stark, K.H Schoenbach, Nanoscond pulsed electric field (nsPEF) effects on cells and tissues: apoptosis induction and tumor growth inhibition, IEEE Trans. Plasma Sci. 30 (2002) 286-292.

[10] S.J. Beebe, P.M. Fox, L.J. Rec, E.L. Willis, K.H. Schoenbach, Nanosecond, high-intensity pulsed electric fields induce apoptosis in human cells, FASEB J. 17 (2003) 1493-1495.

[11] R. Nuccitelli, U. Pliquett, X. Chen, W. Ford, J.R. Swanson, S.J. Beebe, J.F. Kolb, K.H. Schoenbach, Nanosecond pulsed electric fields cause melanomas to self-destruct, Biochem. Biophys. Res. Commun. 343 (2006) 351-360.

[12] E.B. Garon, D. Sawcer, P.T. Vernier, T. Tang, Y. Sun, L. Marcu, M.A. Gundersen, H.P. Koeffler, In vitro and in vivo evaluation and a case report of intense nanosecond pulsed electric field as a local therapy for human malignancies, Int. J. Cancer 121 (2007) 675-682.

[13] M. Stacey, J. Stickley, P. Fox, V. Statler, K.H. Schoenbach, S.J. Beebe, E.S. Buescher, Differential effects in cells exposed to ultra- short, high intensity electric fields: cell survival, DNA damage, and cell cycle analysis, Mutat. Res. 542 (2003) 65-75.

[14] S.J. Beebe, P.F. Blackmore, J. White, R.P. Joshi, K.H. Schoenbach, Nanosecond pulsed electric fields modulate cell function through intracellular signal transduction mechanisms, Physiol. Meas. 25 (2004) 1077-1093.

[15] J.F. Kolb, S. Kono, K.H. Schoenbach, Nanosecond pulsed electric field generators for the study of subcellular effects, Bioelectromagnetics 27 (2006) 172-187.

[16] L.L. Vindelov, Flow microfluorometric analysis of nuclear DNA in cells from solid tumors and suspensions, Virchows Arch. B Cell Pathol. 24 (1977) 227-242.

[17] G. Wang, R. Miskimins, W.K. Miskimins, Mimosine arrests cells in G1 by enhancing the levels of p27 $7^{\mathrm{kip} 1}$, Exp. Cell Res. 254 (1999) 64 71.

[18] J. Teissie, N. Eynard, B. Gabriel, M.P. Rols, Electropermeabilization of cell membranes, Adv. Drug Deliv. Rev. 35 (1999) 3-19.

[19] J.C. Weaver, Yu.A. Chizmadzhev, Theory of electroporation: a review, Bioelectrochem. Bioenerg. 41 (1996) 135-160.

[20] E.A. Lerner, M.R. Lerner, C.A. Janeway Jr., J.A. Steitz, Monoclonal antibodies to nucleic acid-containing cellular constituents: probe for molecular biology and autoimmune disease, Proc. Natl. Acad. Sci. USA 78 (1981) 2737-2741.

[21] G.W. Zieve, R.A. Sauterer, Cell biology of the snRNP particles, Crit. Rev. Biochem. Mol. Biol. 25 (1990) 1-46.

[22] T. Misteli, J.F. Cáceres, D.L. Spector, The dynamics of a pre-mRNA splicing factor in living cells, Nat. 387 (1997) 523-527.

[23] D.L. Spector, W.H. Schrier, H. Busch, Immunoelectron microscopic localization of snRNPs, Biol. Cell 49 (1983) 1-10.

[24] R.T. O'Keefe, A. Mayeda, C.L. Sadowski, A.R. Krainer, D.L. Spector, Disruption of pre-mRNA splicing in vivo results in reorganization of splicing factors, J. Cell Biol. 124 (1994) 249-260. 